J. Korean Math. Soc. 41 (2004), No. 5, pp. 883--894

\title{
CONVERGENCE OF WEIGHTED SUMS FOR DEPENDENT RANDOM VARIABLES
}

\author{
HAN-Ying Liang, Dong-XIA Zhang, AND Jong-Il BaEK
}

\begin{abstract}
We discuss in this paper the strong convergence for weighted sums of negative associated (in abbreviation: NA) arrays. Meanwhile, the central limit theorem for weighted sums of NA variables and linear process based on NA variables is also considered. As corollary, we get the results on iid of $\mathrm{Li}$ et al. ([10]) in NA setting.
\end{abstract}

\section{Introduction}

Many useful linear statistics based on a random sample are weighted sums of i.i.d. random variables. Examples include least-squares estimators, nonparametric regression function estimators and jackknife estimates, among other. In this respect, studies of strong convergence for these weighted sums have demonstrated significant progress in probability theory with applications in mathematical statistics. Up to now, various limit properties for sums of i.i.d. random variables have been studied by many authors.

The most commonly studied method of summation is that of Cesàro's. Set, for $\alpha>-1$,

$$
A_{n}^{\alpha}=\frac{(\alpha+1)(\alpha+2) \cdots(\alpha+n)}{n !} \quad n=1,2, \ldots \text { and } A_{0}^{\alpha}=1 .
$$

Let $\left\{X, X_{n}, n \geq 1\right\}$ be a sequence of i.i.d. random variables. One says that $X$ satisfies Cesàro Law of Large Numbers of order $\alpha, 0<\alpha<1$, if

Received June 11, 2003.

2000 Mathematics Subject Classification: 60F15.

Key words and phrases: strong convergence, weighted sum, Cesàro mean, central limit theorem, negatively associated random variable.

This research was supported by Wonkwang University Grant in 2004. 
and only if

$$
\frac{1}{A_{n}^{\alpha}} \sum_{k=0}^{n} A_{n-k}^{\alpha-1} X_{k} \text { convergences a.s. as } n \rightarrow \infty .
$$

It is well known that

$$
\begin{aligned}
& \lim _{n \rightarrow \infty} \frac{1}{A_{n}^{\alpha}} \sum_{k=0}^{n} A_{n-k}^{\alpha-1} X_{k}=\mu \text { a.s. } \\
& \quad \text { if and only if } E|X|^{1 / \alpha}<\infty \text { and } E X=\mu .
\end{aligned}
$$

For $\alpha=1$ this result is, of course, the classical Kolmogorove strong law. For $1 / 2<\alpha<1$ the proof is due to Lorentz ([14]); for $0<\alpha<1 / 2$ it follows from Chow and Lai ([4]). the case $\alpha=1 / 2$ was treated by Déniel and Derriennic ([5]). Heinkel ([7]) established a version of this result in a Banach space setting. Li et al.([10]) studied the convergence rates of Cesàro Law of Large Numbers and pointed out the following result.

TheOREM 1.1. Let $\left\{X, X_{n}, n \geq 1\right\}$ be a sequence of i.i.d. random variables.

(i) For $0<\alpha<1 / 2$, if $E e^{t|X|}<\infty$ for all $t>0$, then

$$
\frac{1}{A_{n}^{\alpha}} \sum_{k=0}^{n} A_{n-k}^{\alpha-1}\left(X_{k}-E X\right)=o\left(n^{-\alpha} \log n\right) \quad \text { a.s. }
$$

(ii) For $1 / 2<\alpha<1$, if $E(X-E X)^{2}=1$, then

$$
\alpha(2 \alpha-1)^{1 / 2} \Gamma^{2}(\alpha) n^{1 / 2}\left(1 / A_{n}^{\alpha}\right) \sum_{k=0}^{n} A_{n-k}^{\alpha-1}\left(X_{k}-E X\right) \stackrel{\mathcal{D}}{\longrightarrow} N(0,1) .
$$

However, many variables are dependent in actual problems. For example, negatively associated random variables, its definition is as follows:

Definition. A finite family of random variables $\left\{X_{i}, 1 \leq i \leq n\right\}$ is said to be negatively associated (NA) if for every pair of disjoint subsets $A$ and $B$ of $\{1,2, \ldots, n\}$,

$$
\operatorname{Cov}\left(f_{1}\left(X_{i}, i \in A\right), f_{2}\left(X_{j}, j \in B\right)\right) \leq 0
$$

whenever $f_{1}$ and $f_{2}$ are coordinatewise increasing and such that the covariance exists. An infinite family of random variables is NA if every finite subfamily is NA.

This definition is introduced by Alam and Saxena ([1]) and carefully studied by Joag-Dev and Proschan ([8]). As pointed out and proved by Joag-Dev and Proschan ([8]), a number of well known multivariate 
distributions possess the NA property, such as (a) multinomial, (b) convolution of unlike multinomials, (c) multivariate hypergeometric, (d) Dirichlet, (e) Dirichlet compound multinomial, (f) negatively correlated normal distribution, (g) permutation distribution, (h) random sampling without replacement, and (i) joint distribution of ranks. Perhaps, the significance of NA may lie, however, in the perception that NA is the appropriate modeling for several species competing for the same limited resources. Because of its wide applications in multivariate statistical analysis and systems reliability, the notion of NA have received considerable attention recently. we refer to Joag-Dev and Proschan ([8]) for fundamental properties, Matula ([15]) for the three series theorem, $\mathrm{Su}$ et al. ([23]) and Shao ([20]) for moment equalities, Shao and Su ([21]) for law of the iterated logarithm, Liang and Su ([11]) and Liang ([12]) for complete convergence, Roussas ([19]) for the central limit theorem of random fields. In addition, Kim and Baek ([9]) discussed a central limit theorem for linear processes generated by linearly positively quadrantdependent process.

In order to extend Theorem 1.1 to NA setting, in this paper, we will discuss the strong convergence and central limit theorem for weighted sums of NA random variables.

\section{Strong convergence}

Theorem 2.1. Let $\left\{X_{n i}, 1 \leq i \leq k_{n}, n \geq 1\right\}$ be an array of row $N A$ random variables with $E X_{n i}=0$ and

$$
P\left(\left|X_{n i}\right|>x\right)=O(1) P(|X|>x) \text { for all } 1 \leq i \leq k_{n}, n \geq 1 \text { and } x>0,
$$

which $k_{n}, n \geq 1$ is a sequence of positive integers. Assume that $\left\{a_{n i}, 1 \leq\right.$ $\left.i \leq k_{n}, n \geq 1\right\}$ is an array of real numbers satisfying

$$
\text { (i) } \max _{1 \leq i \leq k_{n}}\left|a_{n i}\right|=O\left((\log n)^{-1}\right) \quad \text { (ii) } \sum_{i=1}^{k_{n}} a_{n i}^{2}=o\left((\log n)^{-1}\right) \text {. }
$$

If $E e^{t|X|}<\infty$ for all $t>0$, then

$$
\sum_{n=1}^{\infty} n^{r-2} P\left(\left|\sum_{i=1}^{k_{n}} a_{n i} X_{n i}\right|>\epsilon\right)<\infty \text { for all } \epsilon>0 \text { and all } r \geq 2 .
$$

REMARK 2.1. The following example shows that Theorem 2.1 does not hold if the condition (ii) is replaced by the weaker condition (ii)' $\sum_{i=1}^{k_{n}} a_{n i}^{2}=O\left((\log n)^{-1}\right)$. 
EXAMPLE 1 . Let $\left\{X, X_{i}, i \geq 1\right\}$ be a sequence of iid $N(0,1)$ random variables. Set

$$
a_{n i}= \begin{cases}1 /[\log n], & \text { if } 1 \leq i \leq[\log n] \\ 0, & \text { if }[\log n]+1 \leq i \leq n,\end{cases}
$$

where $[x]$ denotes the integer part of $x$. Then the condition (i) of Theorem 2.1 and the above condition (ii)' are easily satisfied.

Note that $X \sim N(0,1)$, it follows that $E e^{t|X|} \leq 2 e^{t^{2} / 2}$ for all $t>0$. Since $\sum_{i=1}^{[\log n]} X_{i} / \sqrt{[\log n]} \sim N(0,1)$, we have by Lemma 5.1.1 in Stout ([22]) that

$$
\begin{aligned}
P\left(\left|\sum_{i=1}^{n} a_{n i} X_{i}\right|>1\right) & =P\left(\left|\sum_{i=1}^{[\log n]} X_{i} / \sqrt{[\log n]}\right|>\sqrt{[\log n]}\right) \\
& \geq 2 \exp \{-[\log n]\} \geq 2 / n
\end{aligned}
$$

for all sufficiently large $n$, and so

$$
\sum_{n=1}^{\infty} n^{r-2} P\left(\left|\sum_{i=1}^{n} a_{n i} X_{i}\right|>1\right)=\infty \quad \text { for all } r \geq 2,
$$

i.e. Theorem 2.1 does not hold.

Corollary 2.1. Let $\left\{X_{i}, i \geq 0\right\}$ be a sequence of NA random variables and $P\left(\left|X_{i}\right|>x\right)=O(1) P(|X|>x)$ for all $i \geq 0$ and $x>0$. For $0<\alpha<1 / 2$, if $E e^{t|X|}<\infty$ for all $t>0$, then

$$
\frac{1}{A_{n}^{\alpha}} \sum_{k=0}^{n} A_{n-k}^{\alpha-1}\left(X_{k}-E X_{k}\right)=o\left(n^{-\alpha} \log n\right) \quad \text { a.s. }
$$

\section{Central limit theorem}

Theorem 3.1. Let $\left\{X_{i},-\infty<i<\infty\right\}$ be a sequence of mean zero $N A$ random variables satisfying

$$
\sum_{j:|k-j| \geq u}\left|\operatorname{cov}\left(X_{k}, X_{j}\right)\right| \rightarrow 0 \quad \text { as } u \rightarrow \infty \quad \text { uniformly for } k \geq 1 .
$$

Assume that $\left\{a_{n i}, 1 \leq i \leq n, n \geq 1\right\}$ is an array of real numbers satisfying

$$
\sum_{i=1}^{n} a_{n i}^{2}=O(1) \quad \text { and } \quad \max _{1 \leq i \leq n}\left|a_{n i}\right| \rightarrow 0 \text { as } n \rightarrow \infty
$$

and that $\operatorname{Var}\left(\sum_{i=1}^{n} a_{n i} X_{i}\right) \rightarrow 1$. 
(a) If $X_{i}$ is uniformly integrable in $L_{2}$, then

$$
\sum_{i=1}^{n} a_{n i} X_{i} \stackrel{\mathcal{D}}{\longrightarrow} N(0,1)
$$

(b) Put $\xi_{t}=\sum_{j=0}^{\infty} c_{j} X_{t-j}$. Here $\left\{c_{j}\right\}$ is a sequence of real numbers with $C(1)=\sum_{j=0}^{\infty} c_{j} \neq 0$ and $\sum_{j=1}^{\infty} j\left|c_{j}\right|<\infty$. If $X_{i}$ is uniformly integrable in $L_{2}$ and $\max _{1 \leq i \leq n}\left|a_{n i}\right|=O\left(n^{-1 / 2}\right)$, then

$$
\sum_{i=1}^{n} a_{n i} \xi_{i} \stackrel{\mathcal{D}}{\longrightarrow} N\left(0, C^{2}(1)\right)
$$

(c) Put $\eta_{t}=\sum_{j=-\infty}^{\infty} c_{j} X_{t-j}$. Here $\left\{c_{j}\right\}$ is a sequence of real numbers with $D(1)=\sum_{j=-\infty}^{\infty} c_{j} \neq 0$ and $\sum_{j=-\infty}^{\infty} j^{2} c_{j}^{2}<\infty$.-If $\sup _{i} E\left|X_{i}\right|^{2+\delta}<$ $\infty$ for any $\delta>0$ and $\max _{1 \leq i \leq n}\left|a_{n i}\right|=O\left(n^{-1 / 2}\right)$, then

$$
\sum_{i=1}^{n} a_{n i} \eta_{i} \stackrel{\mathcal{D}}{\longrightarrow} N\left(0, D^{2}(1)\right) \text {. }
$$

Corollary 3.1. Let $\left\{X_{i}, i \geq 1\right\}$ be a sequence of NA random variables. For $1 / 2<\alpha<1$, if $X_{i}$ is uniformly integrable in $L_{2}$, then

$$
R_{n}=\alpha(2 \alpha-1)^{1 / 2} \Gamma^{2}(\alpha) n^{1 / 2}\left(1 / A_{n}^{\alpha}\right) \sum_{k=0}^{n} A_{n-k}^{\alpha-1}\left(X_{k}-E X\right) \stackrel{\mathcal{D}}{\longrightarrow} N\left(0, \sigma^{2}\right),
$$

where $\sigma^{2}=\lim _{n \rightarrow \infty} \operatorname{Var} R_{n}$.

REMARK 3.1. In Corollary 3.1 , if $\left\{X, X_{n}, n \geq 1\right\}$ is a sequence of i.i.d. random variables and $E(X-E X)^{2}=1$, then $\sigma^{2}=1$. Since independent r.v.'s are a special case of NA r.v.'s, Corollarys 2.1 and 3.1 extend Theorem 1.1 to NA case.

\section{Proofs of Theorems 2.1 and 3.1}

In this section, $a \ll b$ means $a=O(b), a^{+}=\max (0, a), a^{-}=$ $\max (0,-a)$. Let $C$ and $c$ denote positive constant whose values are unimportant and may vary at different place. 
Proof of Theorem 2.1. Since $a_{n i}=a_{n i}^{+}-a_{n i}^{-}$, it suffices to show

$$
\begin{aligned}
& \sum_{n=1}^{\infty} n^{r-2} P\left(\left|\sum_{i=1}^{k_{n}} a_{n i}^{+} X_{n i}\right|>\epsilon\right)<\infty \quad \text { for any } \epsilon>0, \\
& \sum_{n=1}^{\infty} n^{r-2} P\left(\left|\sum_{i=1}^{k_{n}} a_{n i}^{-} X_{n i}\right|>\epsilon\right)<\infty \quad \text { for any } \epsilon>0 .
\end{aligned}
$$

We prove only (4.3), the proof of (4.4) is analogous. To prove (4.3), we need only to prove

$$
\begin{aligned}
& \sum_{n=1}^{\infty} n^{r-2} P\left(\sum_{i=1}^{k_{n}} a_{n i}^{+} X_{n i}>\epsilon\right)<\infty \quad \text { for any } \epsilon>0, \\
& \sum_{n=1}^{\infty} n^{r-2} P\left(\sum_{i=1}^{k_{n}} a_{n i}^{+} X_{n i}<-\epsilon\right)<\infty \quad \text { for any } \epsilon>0 .
\end{aligned}
$$

We first prove (4.5). From the definition of NA variables, we know that $\left\{a_{n i}^{+} X_{n i}, 1 \leq i \leq k_{n}, n \geq 1\right\}$ is still an arrays of row NA random variables. Noticing $e^{x} \leq 1+x+\frac{1}{2} x^{2} e^{|x|}$ for all $x \in \mathcal{R}$, hence by using lemma 1 of Matula ([15]), we get for $t=M \log n / \epsilon$, where $M$ is a large constant and will be specified later on,

$$
\begin{aligned}
& \sum_{n=1}^{\infty} n^{r-2} P\left(\sum_{i=1}^{k_{n}} a_{n i}^{+} X_{n i}>\epsilon\right) \\
\leq & \sum_{n=1}^{\infty} n^{r-2} e^{-\epsilon t} E e^{t \sum_{i \neq 1}^{k_{n}} a_{n i}^{+} X_{n i}} \\
\leq & \sum_{n=1}^{\infty} n^{r-2-M} \prod_{i=1}^{k_{n}} E e^{t a_{n i}^{+} X_{n i}} \\
\leq & \sum_{n=1}^{\infty} n^{r-2-M} \prod_{i=1}^{k_{n}}\left[1+\frac{1}{2} t^{2}\left(a_{n i}^{+}\right)^{2} E X_{n i}^{2} e^{t a_{n i}^{+}\left|X_{n i}\right|}\right] \\
\ll & \sum_{n=1}^{\infty} n^{r-2-M} \prod_{i=1}^{k_{n}}\left[1+C(\log n)^{2}\left(a_{n i}^{+}\right)^{2} E e^{(1+C)|X|}\right] \\
\leq & \sum_{n=1}^{\infty} n^{r-2-M} \exp \left\{C(\log n)^{2} \sum_{i=1}^{k_{n}}\left(a_{n i}^{+}\right)^{2}\right\}
\end{aligned}
$$




$$
\leq \sum_{n=1}^{\infty} n^{(r+\epsilon)-(2+M)}<\infty
$$

provided $M>(r+\epsilon)-1$. Thus, (4.5) is proved.

By replacing $X_{n i}$ by $-X_{n i}$ from the above statement, and noticing $\left\{a_{n i}^{+}\left(-X_{n i}\right), 1 \leq i \leq k_{n}, n \geq 1\right\}$ is still an arrays of row NA random variables, we know that (4.6) holds.

Lemma 1. (Cai and Roussas ([3])) Let $A$ and $B$ be disjoint subsets of $\mathcal{N}$, and let $\left\{X_{j}, j \in A \cup B\right\}$ be $N A$.

(i) Let $f: \mathcal{R}^{\# A} \rightarrow \mathcal{R}$ and $g: \mathcal{R}^{\# B} \rightarrow \mathcal{R}$ be partially differentiable with bounded partial derivatives, and let $\left\|\partial f / \partial t_{i}\right\|_{\infty}$ stand for the supnorm. Then

$$
\begin{aligned}
& \left|\operatorname{Cov}\left\{f\left(X_{i} ; i \in A\right), g\left(X_{j} ; j \in B\right)\right\}\right| \\
\leq & \sum_{i \in A} \sum_{j \in B}\left\|\frac{\partial f}{\partial t_{i}}\right\|_{\infty} \cdot\left\|\frac{\partial g}{\partial t_{j}}\right\|_{\infty}\left|\operatorname{Cov}\left(X_{i}, X_{j}\right)\right| .
\end{aligned}
$$

(ii) If $q: \mathcal{R} \rightarrow \mathcal{R}$ is a bounded differentiable function with bounded derivative, then

$\left|\operatorname{Cov}\left\{\prod_{i \in A} q\left(X_{i}\right), \prod_{j \in B} q\left(X_{j}\right)\right\}\right| \leq\|q\|_{\infty}^{\# A+\# B-2}\left\|q^{\prime}\right\|_{\infty}^{2} \sum_{i \in A} \sum_{j \in B}\left|\operatorname{Cov}\left(X_{i}, X_{j}\right)\right|$.

Proof of Theorem 3.1. (a) This part has been proved partially in Liang and Jing ([13]), but, for the sake of easy reference, we give a proof here, too. Without loss of generality, we assume that $a_{n i}=0$ for all $i>n$. Note that, for $1 \leq u \leq n-1$

$$
\sum_{i, j=1,|i-j| \geq u}^{n}\left|a_{n i} a_{n j} \operatorname{Cov}\left(X_{i}, X_{j}\right)\right| \leq \sup _{k}\left|\sum_{j,|k-j| \geq u} \operatorname{Cov}\left(X_{i}, X_{j}\right)\right|\left(\sum_{i=1}^{n} a_{n i}^{2}\right),
$$

and hence, by (3.1) and (3.2), for a fixed small $\epsilon>0$, we can find a positive integer $u=u_{\epsilon}$ such that

$$
0 \leq \sum_{i, j=1,|i-j| \geq u}\left|a_{n i} a_{n j} \operatorname{Cov}\left(X_{i}, X_{j}\right)\right| \leq \epsilon
$$


Denote by $[x]$ the integer part of $x$ and define

$$
\begin{aligned}
K & =\left[\frac{1}{\epsilon}\right], \\
Y_{n j} & =\sum_{i=u j+1}^{u(j+1)} a_{n i} X_{i}, j=0,1,2, \ldots \\
A_{j} & =\left\{i: 2 K j \leq i<2 K j+K,\left|\operatorname{Cov}\left(Y_{n i}, Y_{n, i+1}\right)\right|\right. \\
& \left.\leq \frac{2}{K} \sum_{i=2 K j}^{2 K j+K} \operatorname{Var}\left(Y_{n i}\right)\right\} .
\end{aligned}
$$

Since $2\left|\operatorname{Cov}\left(Y_{n i}, Y_{n, i+1}\right)\right| \leq \operatorname{Var}\left(Y_{n i}\right)+\operatorname{Var}\left(Y_{n, i+1}\right)$, we get that for every $j$ the set $A_{j}$ is not empty. Now we define the integers $m_{1}, m_{2}, \ldots, m_{n}$ recurrently by $m_{0}=0$ :

$$
m_{j+1}=\min \left\{m: m>m_{j}, m \in A_{j}\right\}
$$

and put

$$
\begin{aligned}
& Z_{n j}=\sum_{i=m_{j}+1}^{m_{j+1}} Y_{n i}, j=0,1,2, \ldots \\
& \triangle_{j}=\left\{u\left(m_{j}+1\right)+1, \ldots, u\left(m_{j+1}+1\right)\right\} .
\end{aligned}
$$

We observe that

$$
Z_{n j}=\sum_{k \in \triangle_{j}} a_{n k} X_{k}, j=0,1, \ldots
$$

It is easy to see that every set $\triangle_{j}$ contains no more than $3 K u$ elements. Thus, by (3.2), we know that the uniformly integration of $\left\{X_{i}^{2}, i \geq 1\right\}$ implies the uniformly integration of $\left\{Z_{n i}, 1 \leq i \leq n, n \geq 1\right\}$, and hence $\left\{Z_{n i}, 1 \leq i \leq n, n \geq 1\right\}$ satisfies the Lindeberg's Condition. It remains to observe that by Lemma 1 , for any real number $t$

$$
\begin{aligned}
& \left|E \exp \left(i t \sum_{j=1}^{n} Z_{n j}\right)-\prod_{j=1}^{n} E \exp \left(i t Z_{n j}\right)\right| \\
\leq & \left|\operatorname{Cov}\left(\exp \left(i t \sum_{j=1}^{n-1} Z_{n j}\right), \exp \left(i t Z_{n n}\right)\right)\right| \\
+ & \left|E \exp \left(i t Z_{n n}\right)\right|\left|E \exp \left(i t \sum_{j=1}^{n-1} Z_{n j}\right)-\prod_{j=1}^{n-1} E \exp \left(i t Z_{n j}\right)\right|
\end{aligned}
$$




$$
\begin{aligned}
& \ll t^{2} \sum_{1 \leq i<j \leq n}\left|\operatorname{Cov}\left(Z_{n i}, Z_{n j}\right)\right| \\
& =t^{2}\left[\sum_{1 \leq i<j \leq n,|i-j|=1}\left|\operatorname{Cov}\left(Z_{n i}, Z_{n j}\right)\right|+\sum_{1 \leq i<j \leq n,|i-j|>1}\left|\operatorname{Cov}\left(Z_{n i}, Z_{n j}\right)\right|\right] \\
& \ll \quad t^{2}\left[\sum_{1 \leq i<j \leq n,|i-j| \geq u}\left|a_{n i} a_{n j}\right|\left|\operatorname{Cov}\left(X_{i}, X_{j}\right)\right|\right. \\
& \left.\quad+\sum_{j=1}^{n}\left|\operatorname{Cov}\left(Y_{n m_{j}}, Y_{n, m_{j+1}}\right)\right|\right] \\
& \ll \quad t^{2}\left[\epsilon+\frac{O(1)}{K} \sum_{i=1}^{n} \operatorname{Var}\left(Y_{n i}\right)\right] \ll \epsilon t^{2} .
\end{aligned}
$$

Now, Theorem 3.1(a) is proved by Theorem 4.2 in Billingsley ([2]).

(b) Note that

$$
\xi_{k}=C(1) X_{k}+\tilde{X}_{k-1}-\tilde{X}_{k}
$$

where $\tilde{X}_{k}=\sum_{j=0}^{\infty} \tilde{c}_{j} X_{k-j}$ and $\tilde{c}_{j}=\sum_{i=j+1}^{\infty} c_{i}$. Hence

$$
\sum_{i=1}^{n} a_{n i} \xi_{i}=C(1) \sum_{k=1}^{n} a_{n k} X_{k}+\sum_{k=1}^{n} a_{n k}\left(\tilde{X}_{k-1}-\tilde{X}_{k}\right):=I_{n}+J_{n}
$$

By (a), we get $I_{n} \stackrel{\mathcal{D}}{\longrightarrow} N\left(0, C^{2}(1)\right)$.

To prove $J_{n} \stackrel{P}{\rightarrow} 0$, we here state Abel Inequality (see p.32, Theorem 1 of Mitrinovic ([16])):

Let $A_{1}, A_{2}, \ldots, A_{n} ; B_{1}, B_{2}, \ldots, B_{n}\left(B_{1} \geq B_{2} \geq \ldots \geq B_{n} \geq 0\right)$ be two sequences of real numbers, and let $S_{k}=\sum_{i=1}^{k} A_{i}, M_{1}=\min _{1 \leq k \leq n} S_{k}$ and $M_{2}=\max _{1 \leq k \leq n} S_{k}$. Then

$$
B_{1} M_{1} \leq \sum_{k=1}^{n} A_{k} B_{k} \leq B_{1} M_{2}
$$

Without loss of generality, assume that $a_{n 1} \geq a_{n 2} \geq \cdots \geq a_{n n}$. Let $B_{s}=a_{n s}-a_{n n}, 1 \leq s \leq n-1, B_{n}=0$. Applying (4.7) we have

$$
\begin{aligned}
\left|J_{n}\right| & \leq\left|\sum_{k=1}^{n}\left(a_{n k}-a_{n n}\right)\left(\tilde{X}_{k-1}-\tilde{X}_{k}\right)\right|+\left|\sum_{k=1}^{n} a_{n n}\left(\tilde{X}_{k-1}-\tilde{X}_{k}\right)\right| \\
& \leq 2 \max _{1 \leq k \leq n}\left|a_{n k}-a_{n n}\right| \max _{1 \leq m \leq n}\left|\sum_{k=1}^{m}\left(\tilde{X}_{k-1}-\tilde{X}_{k}\right)\right|
\end{aligned}
$$




$$
\begin{aligned}
& +\left|a_{n n}\right|\left|\tilde{X}_{0}-\tilde{X}_{n}\right| \\
\ll & \max _{1 \leq k \leq n}\left|a_{n k}(x)\right|\left(\left|\tilde{X}_{0}\right|+\max _{1 \leq m \leq n}\left|\tilde{X}_{m}\right|\right) \\
= & O\left(n^{-1 / 2}\right)\left(\left|\tilde{X}_{0}\right|+\max _{1 \leq m \leq n}\left|\tilde{X}_{m}\right|\right) .
\end{aligned}
$$

Since $\sum_{j=1}^{\infty} j\left|c_{j}\right|<\infty \Rightarrow \sum_{j=0}^{\infty}\left|\tilde{c}_{j}\right|<\infty$ (see Phillips and Solo ([18]), Lemma 2.1),

$$
E\left|\tilde{X}_{0}\right| \leq \sum_{j=0}^{\infty}\left|\tilde{c}_{j}\right| E\left|X_{-j}\right|<\infty .
$$

On the other hand, observe that

$$
\begin{aligned}
\left|\tilde{X}_{m}\right| & \leq \sum_{i=0}^{m}\left|\tilde{c}_{i}\right|\left|X_{m-i}\right|+\sum_{i=1}^{\infty}\left|\tilde{c}_{m+i}\right|\left|X_{-i}\right| \\
& \leq \max _{0 \leq i \leq m}\left|X_{i}\right|\left(\sum_{i=0}^{m}\left|\tilde{c}_{i}\right|\right)+\sum_{i=1}^{\infty}\left|\hat{c}_{i}\right|\left|X_{-i}\right|
\end{aligned}
$$

with $\hat{c}_{i}=\sum_{j=i+1}^{\infty}\left|c_{j}\right|$. Note that

$$
\sum_{j=1}^{\infty}\left|\tilde{c}_{j}\right| \leq \sum_{j=1}^{\infty} \hat{c}_{j}=\sum_{j=1}^{\infty} \sum_{i=j+1}^{\infty}\left|c_{i}\right| \leq \sum_{j=1}^{\infty} j\left|c_{j}\right|<\infty
$$

and $n^{-1 / 2} \max _{0 \leq m \leq n}\left|X_{m}\right| \stackrel{P}{\rightarrow} 0$ is equivalent to

$$
n^{-1} \sum_{m=0}^{n} X_{m}^{2} I\left(\left|X_{m}\right|>n^{1 / 2} \epsilon\right) \stackrel{P}{\rightarrow} 0, \quad \forall \epsilon>0
$$

(cf. Hall and Heyde ([6]), p.53), which, together with (4.8)-(4.11), follows $J_{n} \stackrel{P}{\rightarrow} 0$.

(c) Note that

$$
\eta_{i}=D(1) X_{i}+\widetilde{X}_{i-1}-\widetilde{X}_{i}+\widetilde{\widetilde{X}}_{i+1}-\widetilde{\widetilde{X}}_{i},
$$

where $\tilde{X}_{i}=\sum_{j=0}^{\infty} \widetilde{c}_{j} X_{i-j}, \quad \widetilde{\widetilde{X}}_{i}=\sum_{j=-\infty}^{0} \widetilde{\widetilde{c}}_{j} X_{i-j}$ and $\widetilde{c}_{j}=\sum_{k=j+1}^{\infty} c_{k}$, $\widetilde{\widetilde{c}}_{j}=\sum_{i=-\infty}^{j-1} c_{i}$.

Similarly to the proof in (b), we need only prove that

$$
n^{-1}\left|\widetilde{X}_{0}\right|^{2}=o_{p}(1), \quad n^{-1} \max _{1 \leq m \leq n}\left|\widetilde{X}_{m}\right|^{2}=o_{p}(1)
$$




$$
n^{-1}\left|\widetilde{\widetilde{X}}_{1}\right|^{2}=o_{p}(1), \quad n^{-1} \max _{1 \leq m \leq n}\left|\widetilde{\widetilde{X}}_{m}\right|^{2}=o_{p}(1)
$$

By $\sum_{j=-\infty}^{\infty} j^{2} c_{j}^{2}<\infty$, we can get $E\left|\widetilde{X}_{0}\right|^{2}<\infty$ and $E\left|\widetilde{\widetilde{X}}_{1}\right|^{2}<\infty$, which follow $n^{-1}\left|\tilde{X}_{0}\right|^{2}=o_{p}(1)$ and $n^{-1}\left|\widetilde{\widetilde{X}}_{1}\right|^{2}=o_{p}(1)$, respectively.

On the other hand, note that

$$
\begin{aligned}
& n^{-1} \max _{1 \leq m \leq n}\left|\widetilde{X}_{m}\right|^{2}=o_{p}(1) \text { if and only if } \\
& \frac{1}{n} \sum_{i=1}^{n} \widetilde{X}_{i}^{2} I\left(\widetilde{X}_{i}^{2}>n c\right)=o_{p}(1) \text { for any } c>0 . \\
& n^{-1} \max _{1 \leq m \leq n}\left|\widetilde{\widetilde{X}}_{m}\right|^{2}=o_{p}(1) \text { if and only if } \\
& \frac{1}{n} \sum_{i=1}^{n} \widetilde{\widetilde{X}}_{i}^{2} I\left(\widetilde{\widetilde{X}}_{i}^{2}>n c\right)=o_{p}(1) \text { for any } c>0
\end{aligned}
$$

(cf. Hall and Heyde ([6]), p.53). Since $\left\{\widetilde{X}_{i}^{2}\right\}$ and $\left\{\widetilde{\widetilde{X}}_{i}^{2}\right\}$ are uniformly integrable by $\sum_{j=-\infty}^{\infty} j^{2} c_{j}^{2}<\infty$ and $\sup _{i} E\left|X_{i}\right|^{2+\delta}<\infty$, by (4.12) and (4.13) we get

$$
n^{-1} \max _{1 \leq m \leq n}\left|\tilde{X}_{m}\right|^{2}=o_{p}(1), \quad n^{-1} \max _{1 \leq m \leq n}\left|\widetilde{\widetilde{X}}_{m}\right|^{2}=o_{p}(1) .
$$

\section{References}

[1] K. Alam and K. M. L. Saxena, Positive dependence in multivariate distributions, Comm. Statist. Theory Methods $\mathbf{A} 10$ (1981), 1183-1196.

[2] P. Billingsley, Convergence of Probability Measures, Wiley, New York, 1968.

[3] Z. W. Cai and G. G. Roussas, Berry-Esseen bounds for smooth estimator of distribution function under association, Nonparametric Statist. 11 (1999), 79-106.

[4] Y. S. Chow and T. L. Lai, Limiting behavior of weighted sums of independent random variables, Ann. Probab. 1 (1973), 810-824.

[5] Y. Déniel and Y. Derriennic, Sur la convergence presque sure, au sens de Cesàro d'ordre $\alpha, 0<\alpha<1$, de variables aléatoires et indépendantes et identiquement distribuées, Probab. Theory Related Fields 79 (1988), 629-636.

[6] P. Hall and C. C. Heyde, Martingale Limit Theory and Its Applications, New York, Academic Press, 1980.

[7] B. Heinkel, An infinite-dimensional law of large numbers in Cesàro's sense, J. Theoret. Probab. 3 (1990), 533-546.

[8] K. Joag-Dev and F. Proschan, Negative association of random variables with applications, Ann. Statist. 11 (1983), 286-295.

[9] T.-S. Kim and J.-I. Baek, A central limit theorem for stationary linear processes generated by linearly positively quadrant dependent process, Statist. Probab. Lett. 51 (2001), 299-305. 
[10] D. L. Li, M. B. Rao, T. F. Jiang and X. C. Wang, Complete convergence and almost sure conver-gence of weighted sums of random variables, J. Theoret. Probab. $8(1995), 49-76$.

[11] H. Y. Liang and C. Su, Complete convergence for weighted sums of NA sequences, Statist. Probab. Lett. 45 (1999), 85-95.

[12] H. Y. Liang, Complete convergence for weighted sums of negatively associated random variables, Statist. Probab. Lett. 48 (2000), 317-325.

[13] H. Y. Liang and B. Y. Jing, Asymptotic properties for estimates of nonparametric regression models based on negatively associated sequences, Submitted, 2002.

[14] G. G. Lorentz, Borel and Banach properties of methods of summation, Duke Math. J. 22 (1955), 129-141.

[15] P. Matula, A note on the almost sure convergence of sums of negatively dependences random variables, Statist. Probab. Lett. 15 (1992), 209-213.

[16] D. S. Mitrinovic, Analytic Inequalities, New York, Springer, 1970.

[17] M. Peligrad and S. Utev, Central limit theorem for linear processes, Ann. Probab. 25 (1997), no. 1, 443-456.

[18] P. C. B. Phillips and V. Solo, Asymptotics for linear processes, Ann. Statist. 20 (1992), 971-1001.

[19] G. G. Roussas, Asymptotic normality of random fields of positively or negatively associated processes, J. Multivariate Anal. 50 (1994), 152-173.

[20] Q. M. Shao, A comparison theorem on maximum inequalities between negatively associated and independent random variables, J. Theoret. Probab. 13 (2000), 343356.

[21] Q. M. Shao and $\mathrm{C}$. Su, The law of the iterated logarithm for negatively associated random variables, Stochastic Process. Appl. 83 (1999), 139-148.

[22] W. F. Stout, Almost Sure Convergence, Academic Press, New York, 1974.

[23] C. Su, L. C. Zhao and Y. B. Wang, Moment inequalities and week convergence for negatively associated sequences, Sci. China Ser. A 40 (1997), 172-182.

Han-Ying Liang and Dong-Xia Zhang

Department of Applied Mathematics

Tongji University

Shanghai 200092, P. R. China

E-mail: hyliang83@hotmail.com

Jong-Il Baek

School of Mathematics \& Informational Statistics

and Institute of Basic Natural Science

Wonkwang University

Ik-San 570-749, Korea

E-mail: jibaek@wonkwang.ac.kr . 